\title{
Efectos depresivos del aislamiento preventivo obligatorio asociados a la pandemia del Covid-19 en docentes y estudiantes de una universidad pública en Colombia
}

\author{
Depressive effects of compulsory preventive isolation \\ associated with the Covid-19 pandemic on teachers and \\ students of a public university in Colombia
}

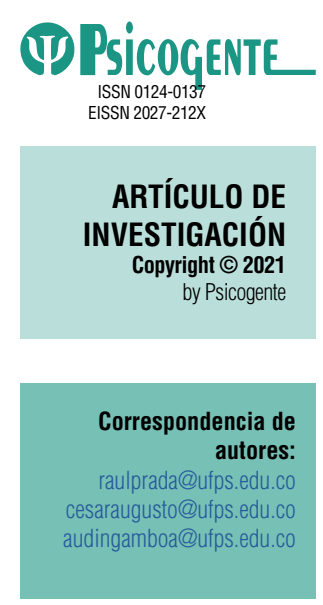

Recibido: $11-05-2020$ Aceptado: 08-10-2020 Publicado: 01-01-2021

\section{Efeitos depressivos do isolamento preventivo obrigatório} associado à pandemia de Covid-19 nos professores e estudantes de uma universidade pública na Colômbia

\author{
Raúl Prada Núñez iD - Audin Aloiso Gamboa Suárez (iD - \\ César Augusto Hernández Suárez iD \\ Universidad Francisco de Paula Santander, Cúcuta, Colombia
}

Resumen

Objetivo: Determinar los efectos depresivos causados por el aislamiento preventivo por la pandemia de Covid-19 en estudiantes y docentes universitarios.

Método: Enfoque cuantitativo a nivel descriptivo-correlacional. Se aplicó el inventario de depresión de Beck a una muestra de 100 docentes y 394 estudiantes de una universidad pública en Colombia.

Resultados: En cuanto a los niveles de afectación no hay diferencias significativas entre docentes y estudiantes, sin embargo, sí hay diferencias respecto al género, con mayor incidencia en los hombres; así mismo se identificó que las personas entre 16 y 35 años se ubican en los niveles de depresión moderada y grave. Para el estado civil se determinó con mayor presencia en la depresión mínima, contrario a las personas viudas que se concentran en el nivel de depresión grave.

Conclusión: Para las personas encuestadas se presentó algún nivel de afectación, lo que se convierte en un antecedente de los posibles efectos del aislamiento preventivo obligatorio. Los resultados del estudio son relevantes para la generación de programas de intervención en bienestar universitario que mitiguen el efecto de esta situación de anormalidad social. Dado que la muestra no es probabilística no se pueden hacer inferencias, sin embargo, los resultados reflejan la existencia de afectaciones en un grupo considerable de personas que no pueden ser deslegitimadas.

Palabras clave: aislamiento social; actores educativos; depresión; Covid-19; educación superior.

Abstract

Objective: To determine the depressive effects caused by preventive isolation by the Covid-19 pandemic on university students and teachers.

Methods: Quantitative approach at the descriptive-correlational level. Beck's depression inventory was applied to a sample of 100 teachers and 394 students of a public university in Colombia.

Results: There were no significant differences between teachers and students in terms of the levels of affectation, but there were differences with respect to gender, with a greater incidence in men; likewise,

Cómo citar este artículo (APA):

Prada Núñez, R., Gamboa Suárez, A.A. \& Hernández Suárez, C.A. (2021). Efectos depresivos del aislamiento preventivo obligatorio asociados a la pandemia del Covid-19 en docentes y estudiantes de una universidad pública en Colombia. Psicogente 24(45), 1-20. https://doi.org/10.17081/ psico.24.45.4156 
it was identified that people between 16 and 35 years old are located in the levels of moderate and severe depression. Marital status was determined to be more prevalent in minimal depression, as opposed to widows who are concentrated in severe depression

Conclusion: For the people surveyed, there was some level of affectation, which becomes a background of the possible effects of compulsory preventive isolation. The results of the study are relevant for the generation of intervention programs in university welfare that mitigate the effect of this situation of social abnormality. Since the sample is not probabilistic, no inferences can be made, however, the results reflect the existence of affectations in a considerable group of people that cannot be delegitimized.

Keywords: social isolation; educational actors; depression; Covid-19; higher education.

Resumo

Objectivo: Determinar os efeitos depressivos causados pelo isolamento preventivo pela pandemia de Covid-19 em estudantes universitários e professores.

Métodos: Abordagem quantitativa a nível descritivo-correlacional. O inventário de depressão de Beck foi aplicado a uma amostra de 100 professores e 394 estudantes de uma universidade pública na Colômbia.

Resultados: Não houve diferenças significativas entre professores e estudantes em termos dos níveis de afecção, mas houve diferenças no que diz respeito ao género, com maior incidência nos homens; do mesmo modo, foi identificado que as pessoas entre os 16 e 35 anos de idade estão localizadas nos níveis de depressão moderada e grave. O estado civil foi determinado como sendo mais prevalecente em depressão mínima, ao contrário das viúvas que se concentram em depressão severa.

Conclusão: Para as pessoas inquiridas, houve algum nível de afetação, que se torna um pano de fundo dos possíveis efeitos do isolamento preventivo obrigatório. Os resultados do estudo são relevantes para a geração de programas de intervenção no bem-estar universitário que atenuem o efeito desta situação de anormalidade social. Uma vez que a amostra não é probabilística, não se podem fazer inferências, no entanto, os resultados reflectem a existência de afecções num grupo considerável de pessoas que não podem ser deslegitimadas.

Palavras-chave: isolamento social; actores educativos; depressão; Covid-19; ensino superior.

\section{INTRODUCCIÓN}

En el mes de diciembre de 2019 en la ciudad de Wuhan, provincia de Hubei en China, surgió un virus denominado SARS-Cov-2 que produce la enfermedad Ilamada Covid-19, (Gorbalenya, Baker, Baric, de Groot, Drosten, Gulyaeva, Haagmans, Lauber, Leontovich, Neuman, Penzar, Perlman, Poon, Samborskiy, Sidorov, Sola, \& Ziebuhr, 2020). Este virus según estudios realizados produce enfermedades respiratorias, entéricas, hepáticas y neurológicas (Guzmán-Del Giudice, Lucchesi-Vásquez, Trelles-De Belaúnde, Pinedo-Gonzales, CamereTorrealva, Daly y Pichilingue-Chagray, 2020; Aragón, Vargas y Miranda, 2019 y Ríos-González, 2019), además presenta un "síndrome de respuesta inflamatoria sistémica, que puede conducir a un choque séptico con fallo multiorgánico, con altas tasas de mortalidad" (Ruiz y Jiménez, 2020, p.33).

El 30 de enero de 2020 la Organización Mundial de la Salud OMS (2020), declara la emergencia sanitaria de preocupación internacional, argumentando los efectos negativos que el virus podría tener en países con menor desarrollo y estructura hospitalaria deficiente. Según la misma organización el nuevo coronavirus SARS-Cov-2, ha infectado a más de tres millones de personas, con una alarmante cifra de 214.000 muertos y 893.000 recuperados en todo el mundo. En Colombia particularmente el virus, según el Instituto 
Nacional de Salud (INSALUD, 2020) muestra cifras también preocupantes, a fecha del 27 de abril de 2020 en el país hay 5.597 casos confirmados, 253 muertes y 1.210 personas recuperadas.

Este brote activó las alarmas del Estado colombiano y el 22 de marzo de 2020 se emitió el Decreto 457, mediante el cual se imparten instrucciones para el cumplimiento del aislamiento social preventivo obligatorio en todo el territorio colombiano. El aislamiento social o la ausencia de relaciones sociales satisfactorias y de un nivel bajo de participación en la vida comunitaria (Burgos, 2014) es un tema de gran interés en la comunidad académica, sobre todo en población de características particulares como los pacientes crónicos (Stefani, Seidmann, Pano, Acrich y Bail, 2003) y los adultos mayores (PabónPoches, 2019). Sin embargo, son escasas las investigaciones que abordan el tema de aislamiento social en toda la población o en actores educativos.

Esta medida del Gobierno nacional cobijó también a instituciones educativas de todos los niveles, y se definió por gran parte de las directivas educativas la virtualización de los procesos de formación. En este sentido, la virtualización apresurada obligada por la pandemia del Covid-19, generó una serie de reflexiones y estrategias de acompañamiento desde la educación virtual, pero también visibilizó la falta de preparación que tienen las universidades de enfoque presencial en Colombia para enfrentar procesos de virtualización (Lara, 2009), y surgieron fenómenos ya antes advertidos, como los problemas de conectividad en poblaciones de contextos rurales (Sánchez, Ramírez y Cañón, 2019), la escases de dispositivos para los estudiantes de bajos recursos (Rama, 2019) y la falta de preparación de los profesores en el manejo de tecnologías digitales (Rangel y Peñalosa, 2013; Martín, Hernández y Mendoza, 2017), entre otros.

Estas deficiencias descritas demuestran un complejo fenómeno que evidencia la escasa apropiación de las tecnologías digitales por parte de la institución y los actores educativos que dificultan la implementación de las TIC en las actividades académicas sobre todo en tiempos de aislamiento por la pandemia. De este modo, no solo las dificultades de uso y apropiación de las tecnologías digitales afectan los actores educativos, hay otros elementos de corte psicológico que pueden obstaculizar los procesos formativos y de enseñanza, como los efectos depresivos relacionados con la falta de sueño, ansiedad y autoestima (Almonte, Parra y Baltazar, 2019) que posiblemente sean generados por el aislamiento preventivo y que derivan en fracaso 
escolar y posteriormente al abandono de los escenarios escolares (Ovalles, Urbina y Gamboa, 2014; Gamboa, 2016).

Es importante señalar que este estudio tiene una relevancia importante, puesto que las instituciones de educación superior requieren de una lectura inicial de los efectos psicológicos del aislamiento obligatorio en los actores educativos y de esta manera generar planes de intervención para disminuirlos. Del mismo modo, se puede evidenciar que son escasas las investigaciones recientes relacionadas con dichos efectos, en este sentido este estudio contribuye a la construcción de conocimiento desde el campo de la psicología a este reciente fenómeno social.

\section{MÉTODO}

\subsection{Diseño empleado}

Esta investigación está enmarcada en el enfoque cuantitativo-descriptivo-correlacional, puesto que pretendió caracterizar un grupo particular de población, por medio de categorías y descripciones de estas (Prada, Hernández y Gamboa, 2019a y Prada, Hernández y Gamboa, 2019b). Desde esta perspectiva, se trata de una investigación de campo, por cuanto la información fue recolectada de la fuente primaria que en este caso fueron docentes y estudiantes.

\subsection{Participantes}

La población que hizo parte de este estudio corresponde a docentes y estudiantes vinculados a los diversos programas de pregrado (Derecho, Trabajo Social, Comunicación Social, Arquitectura y Licenciaturas en Matemáticas, en Educación Infantil y en Ciencias Naturales y Educación Ambiental) de la Facultad de Educación, Artes y Humanidades en la Universidad Francisco de Paula Santander - UFPS, Institución de Educación Superior pública ubicada en Cúcuta, nororiente de Colombia.

Mediante la aplicación del muestreo no probabilístico bajo la técnica de muestreo por cuotas, se conformó los participantes del estudio integrado por 100 docentes y 394 estudiantes quienes voluntariamente accedieron a diligenciar la totalidad del instrumento el cual se puso a disposición en su versión en línea, siendo este el criterio de inclusión. Con relación al perfil demográfico (ver Tabla 1) de los encuestados, se identificó predominio del género femenino en una razón de tres a uno. Con respecto a la edad se 
observa que aproximadamente el $40 \%$ de ellos tienen edades que oscilan entre 16 y 25 años que corresponden a la mayor concentración de estudiantes integrantes de la muestra. Con relación a los otros grupos etarios, se resalta que aproximadamente el $52 \%$ tienen edades que oscilan entre los 26 y los 45 años, el cual correspondería a una buena parte de los estudiantes ( $30 \%$ ) y un $22 \%$ de los docentes encargados de orientar las diversas asignaturas en cada programa académico, el porcentaje restante que se asocia a los informantes con edad mayor a 45 años corresponde al restante del grupo docente. En lo concerniente con el estado civil de los encuestados, se determinó que el $49 \%$ son solteros, mientras que en el $46 \%$ de los casos se observa la existencia de una relación formal con su cónyuge (casados) o informal (unión libre). Finalmente, aproximadamente el $80 \%$ de los encuestados antes de decretarse el aislamiento preventivo obligatorio, tenían como actividad principal estudiar mientras que el porcentaje restante lo representan los docentes.

Tabla 1.

Perfil demográfico de los informantes

\begin{tabular}{|c|c|c|c|}
\hline CARACTERÍSTICA & OPCIONES DE RESPUESTA & FRECUENCIA & PORCENTAJE \\
\hline \multirow{3}{*}{ Género } & Femenino & 366 & 74,1 \\
\hline & Masculino & 128 & 25,9 \\
\hline & Total & 494 & 100,0 \\
\hline \multirow{6}{*}{$\begin{array}{l}\text { Rango de edad } \\
\text { (años) }\end{array}$} & Entre 16 y 25 & 196 & 39,7 \\
\hline & Entre 26 y 35 & 153 & 31,0 \\
\hline & Entre 36 y 45 & 103 & 20,9 \\
\hline & Entre 46 y 55 & 31 & 6,3 \\
\hline & Más de 55 & 11 & 2,2 \\
\hline & Total & 494 & 100,0 \\
\hline \multirow{8}{*}{ Estado civil } & Soltero(a) & 242 & 49,0 \\
\hline & Casado(a) & 107 & 21,7 \\
\hline & Separado(a) & 19 & 3,8 \\
\hline & Unión libre & 121 & 24,5 \\
\hline & Viudo(a) & 1 & 0,2 \\
\hline & Otra & 4 & 0,8 \\
\hline & Total & 494 & 100,0 \\
\hline & Título Media Técnica & 394 & 79,7 \\
\hline \multirow{4}{*}{ Máximo nivel de escolaridad } & Profesional universitario & 15 & 3,0 \\
\hline & Especialización & 57 & 11,5 \\
\hline & Maestría & 25 & 5,1 \\
\hline & Doctorado & 3 & 0,1 \\
\hline \multirow{4}{*}{$\begin{array}{l}\text { Actividad principal que } \\
\text { realizaba }\end{array}$} & Total & 494 & 100,0 \\
\hline & Estudiar & 394 & 79,7 \\
\hline & Trabajar & 100 & 20,3 \\
\hline & Total & 494 & 100,0 \\
\hline
\end{tabular}




\subsection{Instrumento}

Se recurre a la encuesta como instrumento de recolección de datos ya que "cuando se trata de lograr una caracterización-diagnóstico que permita diseñar estrategias de intervención en una comunidad" (Falcón, Pertile y Ponce, 2019, p.1). El instrumento diseñado para este estudio se compone de dos secciones que se detallan en Tabla 2. La primera sección contiene una serie de características asociadas al perfil demográfico del informante las cuales ofrecían diversas opciones con una única respuesta. En la segunda sección se listan los 21 ítems que corresponden al Inventario de Depresión de Beck (BDI-2) el cual ofrece cuatro niveles de calificación de cada uno de los sentimientos o emociones que se hubiesen presentado en ellos en las últimas dos semanas, en el que se ha sentido con más rigor el aislamiento obligatorio dado que corresponde a la ventana de tiempo asociada entre los 30 y 45 días después de haber iniciado esta medida preventiva.

Tabla 2.

Composición del instrumento

\begin{tabular}{|c|c|c|}
\hline & SECCIÓN & DESCRIPCIÓN \\
\hline 1 & Perfil demográfico & $\begin{array}{l}\text { Género, edad, estado civil, máximo nivel de escolaridad, actividad principal desarrollada } \\
\text { antes de la cuarentena. }\end{array}$ \\
\hline 2 & Inventario de Depresión de Beck & $\begin{array}{c}\text { Tristeza, pesimismo, fracaso, pérdida de placer, sentimientos de culpa, sentimientos de cas- } \\
\text { tigo, disconformidad con uno mismo, autocrítica, pensamientos suicidas, llanto, agitación, } \\
\text { pérdida de interés, indecisión, desvalorización, pérdida de energía, cambios en los hábitos } \\
\text { de sueño, irritabilidad, cambios de apetito, dificultad de concentración, cansancio y pérdida } \\
\text { de interés en el sexo. }\end{array}$ \\
\hline
\end{tabular}

En cada ítem se definían cuatro niveles de respuesta cuyos valores oscilaban entre cero (0) y tres (3), luego al final del instrumento se puede calcular para cada participante la puntuación total alcanzada en el BDI-2 apoyado en las puntuaciones de corte, se definen las diferentes categorías o niveles de gravedad de la sintomatología depresiva (Beck, Steer \& Brown, 1996, p.11):

a) Depresión Mínima correspondiente a puntuaciones entre 0 y 13 puntos;

b) Depresión Leve correspondiente a puntuaciones entre 14 y 19 puntos; c) Depresión Moderada para puntuaciones entre 20 y 28 puntos; finalmente, d) Depresión Grave correspondiente a puntuaciones entre 29 y 63 puntos.

\subsection{Análisis de datos}

Una vez se contó con los datos se procedió a exportarlos al SPSS v25 en donde se procesaron de forma descriptiva después de haber configurado la data. Se recurrió a la determinación de estadísticos (frecuencias, porcentajes, p-valor, entre otros) que apoyaron la caracterización de los informantes en cuanto a su nivel de depresión. 


\subsection{Procedimiento}

Ante el aislamiento obligatorio decretado desde el gobierno central, de forma abrupta los habitantes del país han tenido que cambiar sus hábitos de vida, aspecto que posiblemente genera afectaciones emocionales, por tal motivo surge esta necesidad de investigación con el fin de determinar el nivel de afectación en docentes y estudiantes vinculados durante el primer semestre del $\mathbf{2 0 2 0}$ a diversos programas académicos ofertados en la universidad. Para lo cual se identificó el Inventario de Depresión de Beck (BDI-2) como el instrumento idóneo para tal fin a partir de la consulta con profesionales expertos en el tema.

El cuestionario aplicado se incorporó a una encuesta en la que se incluyó una sección inicial cuyo objetivo era la caracterización del perfil demográfico del informante. Definido el instrumento se procedió a crear un formulario en Google. Inicialmente se envió un correo a la totalidad de docentes y estudiantes de la facultad en la que se les notificaba de la investigación y se les invitaba a participar como informantes en ella. Si aceptaban debían responder este correo manifestando su voluntad. De esa forma se consolidó una muestra de docentes y estudiantes quienes recibieron el link del instrumento. Pasadas las dos semanas definidas para la recolección de los datos, estos fueron descargados de la nube en un archivo de Excel, en el cual se codificaron las opciones de respuesta en cada uno de los ítems, para ser exportados los datos al SPSS v25. De esa forma se construyó la data, a partir de la cual se procesaron inicialmente los datos demográficos de los informantes. Posteriormente, se calculó la puntuación de cada informante (sumatoria de la totalidad de los ítems) respecto al BDI-2, según se define en Beck, Steer \& Brown (1996); luego, a partir de este valor se generó una nueva variable de tipo cualitativa (que define los niveles de depresión mencionados en la sección 2.3 de este documento) que permitía identificar el nivel de afectación emocional del informante según la escala implementada. Con esta nueva variable se determinó el porcentaje de informantes en cada nivel de depresión y posteriormente, mediante la selección de casos se analizó en detalle el porcentaje de afectación de los informantes en cada uno de los ítems evaluados en BDI-2. Inicialmente se pensó que podrían existir diferencias en los resultados entre los grupos de docentes y estudiantes, pero después de verificar la normalidad de los datos, se aplicó una prueba ANOVA y se determinó que no existían diferencias significativas en ellos (ver Tabla 3) con un nivel de significancia del $5 \%$, por tal motivo se procesaron de forma conjunta. 
Tabla 3.

Análisis de Varianza para los Niveles de depresión considerando diferencias entre los dos grupos de informantes

\begin{tabular}{cccccc}
\hline & SUMA DE CUADRADOS & GL & $\begin{array}{c}\text { MEDIA } \\
\text { CUADRÁTICA }\end{array}$ & F & SIG. \\
\hline Entre grupos & 11,492 & 1 & 11,492 & 2,10 & 0,1479 \\
Dentro de grupos & 2690,716 & 492 & 5,47 & & \\
Total & 281,209 & 493 & & & \\
\hline
\end{tabular}

\section{RESULTADOS}

Tal como se afirma en Beck, Steer \& Brown (2009), el siglo XXI se ha empezado a considerar como la era de la melancolía debido al número de trabajos que han identificado el aumento de los trastornos depresivos en las personas. Dicho trastorno adopta diversas manifestaciones que están condicionadas por las características sociales y culturales, que podrían presentarse como tristeza, desinterés, desaliento y una imparable sensación de desesperanza. En el manual original del BDI-2 (Beck, Steer \& Brown, 1996) se proponen las diferentes categorías o niveles de depresión.

En la Figura 1 se puede visualizar la distribución porcentual de los encuestados dentro de los cuatro niveles de depresión, resaltando que, en opinión de los informantes y derivado del proceso de aislamiento obligatorio, el $76 \%$ de ellos consideran que están experimentando síntomas de depresión mínima seguida del 14 \% quienes se ubican en el nivel de depresión leve. Se destaca que el $3 \%$ de ellos, afirman estar experimentando síntomas de depresión grave lo cual se convierte una situación de especial interés.

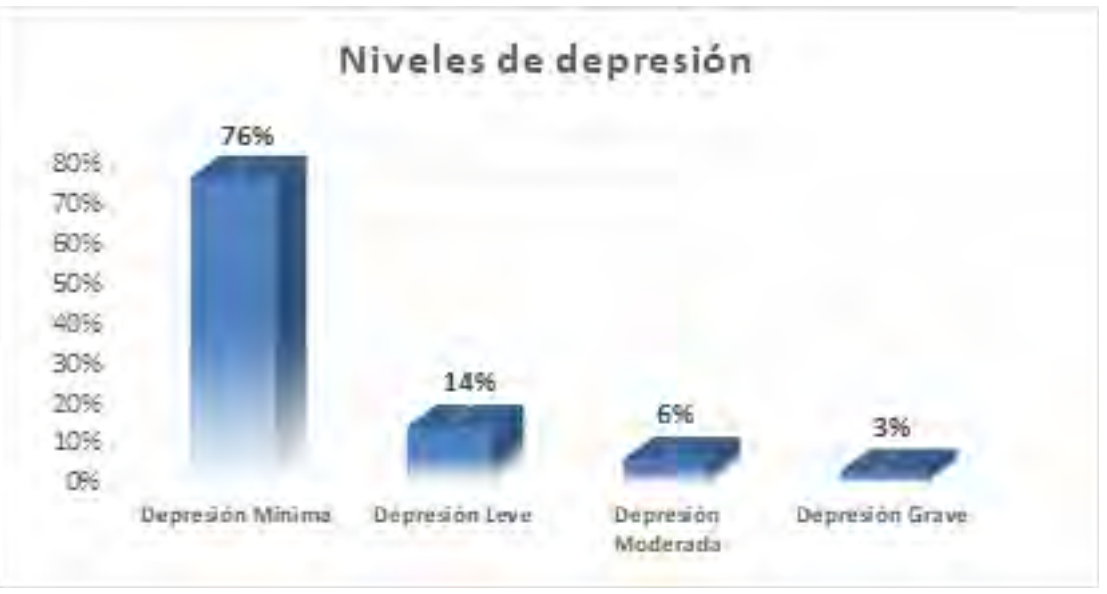

Figura 1. Comparativo de los diversos niveles de depresión encontrados en la muestra 
A continuación, se realiza un análisis detallado de las afectaciones manifestadas por los informantes en cada uno de los niveles de depresión. Para proporcionar simplicidad en la interpretación de los resultados se realizó el siguiente tratamiento en cada uno de los ítems considerados en la escala BDI-2. Para cada sentimiento se ofrecen cuatro opciones de respuesta, de las cuales la primera opción (numerada con 0) se asocia con el hecho de que no hay afectación en ese aspecto durante el período de medición, por ello a esta opción se le asocia con una nueva categoría designada como "No afectado"; mientras que en las opciones siguientes (numeradas del 1 al 3) se evidencia algún tipo de afectación en esta época de cuarentena, por tal motivo estos tres niveles se agrupan en una nueva categoría llamada "Afectado".

\subsection{Características asociadas al nivel de depresión mínima}

Del grupo de encuestados se identificó que 377 (equivalente al $76 \%$ del total) manifestaron tener síntomas asociados a este nivel, destacándose (los porcentajes referidos en cada sentimiento se calculan en función del total de informantes en este nivel, es decir, 377 personas) los porcentajes en las dos categorías ya descritas (ver Tabla 4).

Tabla 4.

Descripción de los indicadores de la escala en el nivel de depresión mínima

\begin{tabular}{ccc}
\hline ASPECTO EVALUADO & NO AFECTADO & AFECTADO \\
\hline Tristeza & $54 \%$ & $46 \%$ \\
Pesimismo & $82 \%$ & $18 \%$ \\
Fracaso & $95 \%$ & $5 \%$ \\
Pérdida de placer & $62 \%$ & $38 \%$ \\
Sentimientos de culpa & $84 \%$ & $16 \%$ \\
Sentimiento de castigo & $93 \%$ & $7 \%$ \\
Disconformidad con uno mismo & $94 \%$ & $6 \%$ \\
Autocrítica & $82 \%$ & $8 \%$ \\
Pensamientos o deseos suicidas & $97 \%$ & $3 \%$ \\
Llanto & $89 \%$ & $11 \%$ \\
Agitación & $68 \%$ & $32 \%$ \\
Pérdida de interés & $81 \%$ & $19 \%$ \\
Indecisión & $84 \%$ & $16 \%$ \\
Desvalorización & $95 \%$ & $5 \%$ \\
Pérdida de energía & $65 \%$ & $35 \%$ \\
Cambio de hábitos de sueño & $33 \%$ & $67 \%$ \\
Irritabilidad & $77 \%$ & $23 \%$ \\
Cambios en el apetito & $53 \%$ & $47 \%$ \\
Dificultad de concentración & $71 \%$ & $29 \%$ \\
Cansancio o fatiga & $73 \%$ & $27 \%$ \\
Pérdida de interés en el sexo & $88 \%$ & $12 \%$ \\
\hline & &
\end{tabular}


A partir de la información, se pudo identificar en este grupo de encuestados que ellos atribuyen como efectos derivados del aislamiento social preventivo, el experimentar: aumento de la tristeza en mucho más momentos del día, no disfrutar en la misma medida de las cosas como lo sentía antes, sentirse más inquieto; lo que desencadena mayor tensión, sensación de cansancio más frecuentemente y mayor dificultad para mantenerse concentrado en el desarrollo de actividades; pero los rasgos más representativos en este grupo son la pérdida de sueño en el $39 \%$ y el aumento del apetito en el $29 \%$ de los casos.

\subsection{Características asociadas al nivel de depresión leve}

En los participantes se logró identificar que 68 de ellos (equivalente al $14 \%$ del total) manifestaron tener afectaciones que los ubican en este nivel. En la Tabla 5 se detallan los porcentajes asociados a cada una de las categorías de análisis.

Tabla 5.

Descripción de los indicadores de la escala en el nivel de depresión leve

\begin{tabular}{ccc}
\hline ASPECTO EVALUADO & NO AFECTADO & AFECTADO \\
\hline Tristeza & $6 \%$ & $94 \%$ \\
Pesimismo & $41 \%$ & $59 \%$ \\
Fracaso & $75 \%$ & $25 \%$ \\
Pérdida de placer & $13 \%$ & $87 \%$ \\
Sentimientos de culpa & $56 \%$ & $44 \%$ \\
Sentimiento de castigo & $75 \%$ & $25 \%$ \\
Disconformidad con uno mismo & $60 \%$ & $40 \%$ \\
Autocrítica & $48 \%$ & $52 \%$ \\
Pensamientos o deseos suicidas & $82 \%$ & $18 \%$ \\
Llanto & $53 \%$ & $47 \%$ \\
Agitación & $28 \%$ & $72 \%$ \\
Pérdida de interés & $27 \%$ & $73 \%$ \\
Indecisión & $37 \%$ & $63 \%$ \\
Desvalorización & $71 \%$ & $29 \%$ \\
Pérdida de energía & $9 \%$ & $91 \%$ \\
Cambio de hábitos de sueño & $4 \%$ & $96 \%$ \\
Irritabilidad & $25 \%$ & $75 \%$ \\
Cambios en el apetito & $19 \%$ & $81 \%$ \\
Dificultad de concentración & $18 \%$ & $82 \%$ \\
Cansancio o fatiga & $31 \%$ & $69 \%$ \\
Pérdida de interés en el sexo & $50 \%$ & $50 \%$ \\
\hline
\end{tabular}

A partir de los resultados mostrados se lograron identificar algunos efectos derivados del aislamiento social, tales como incremento del desaliento respecto a su futuro que viene acompañado de la pérdida de placer e interés 
por las cosas que solían disfrutar; que sumado al aumento de las dudas al momento de tomar decisiones, mayor sensación de cansancio físico e irritabilidad, junto con sentimientos de culpa, que en algunas ocasiones han producido llanto como medio de expresión, terminan generando un panorama complejo.

Adicionalmente, como aspectos de mayor impacto se encuentran en al menos el $80 \%$ de ellos que la tristeza ha empezado a estar presente en mucho más tiempo del día, acompañado de la pérdida de energía la cual compensa con el incremento en las horas de sueño, dificultad para mantenerse concentrado en el desarrollo de una tarea y finalmente desórdenes alimenticios manifestados principalmente en aumento del apetito.

\subsection{Características asociadas al nivel de depresión moderada}

Del grupo de encuestados se identificó que 30 de ellos (equivalente al $6 \%$ del total) manifestaron tener afectaciones que los ubican en este nivel. Los porcentajes asociados a cada una de las categorías de análisis se pueden ver en la Tabla 6.

Tabla 6.

Descripción de los indicadores de la escala en el nivel de depresión moderada

\begin{tabular}{ccc}
\hline ASPECTO EVALUADO & NO AFECTADO & AFECTADO \\
\hline Tristeza & $9 \%$ & $91 \%$ \\
Pesimismo & $13 \%$ & $87 \%$ \\
Fracaso & $38 \%$ & $62 \%$ \\
Pérdida de placer & $9 \%$ & $91 \%$ \\
Sentimientos de culpa & $19 \%$ & $81 \%$ \\
Sentimiento de castigo & $47 \%$ & $53 \%$ \\
Disconformidad con uno mismo & $22 \%$ & $78 \%$ \\
Autocrítica & $22 \%$ & $78 \%$ \\
Pensamientos o deseos suicidas & $66 \%$ & $34 \%$ \\
Llanto & $47 \%$ & $53 \%$ \\
Agitación & $16 \%$ & $84 \%$ \\
Pérdida de interés & $16 \%$ & $84 \%$ \\
Indecisión & $19 \%$ & $81 \%$ \\
Desvalorización & $31 \%$ & $69 \%$ \\
Pérdida de energía & $6 \%$ & $94 \%$ \\
Cambio de hábitos de sueño & $6 \%$ & $94 \%$ \\
Irritabilidad & $19 \%$ & $81 \%$ \\
Cambios en el apetito & $6 \%$ & $94 \%$ \\
Dificultad de concentración & $3 \%$ & $97 \%$ \\
Cansancio o fatiga & $19 \%$ & $81 \%$ \\
Pérdida de interés en el sexo & $56 \%$ & $44 \%$ \\
\hline & &
\end{tabular}


En la Tabla 6 se identifica que al menos en el $50 \%$ de los encuestados ubicados en esta categoría vieron afectados todos los aspectos analizados en la escala de Beck con excepción de la presencia de deseos suicidas y la pérdida del interés hacía el sexo. Asimismo, manifiestan sentirse fracasados, llevándolos al punto de llorar (esto se evidencia en el 53 \% de los casos). Se destacan otras afectaciones adicionales como el aumento del pesimismo, sentimientos de culpa, pérdida de interés, indecisión, irritabilidad y cansancio físico; pero en al menos el $90 \%$ de los encuestados ubicados en este nivel se ha identificado la fuerte presencia de sentimientos de tristeza, pérdida de placer, concentración y energía junto con una reducción del sueño que termina propiciando cambios en el apetito los cuales se han manifestado en los dos casos extremos, por un lado el aumento en la ingesta de alimentos mientras que en otros, ha sido la pérdida del interés por el consumo.

\subsection{Características asociadas al nivel de depresión grave}

En los participantes del estudio se evidenció que 17 de ellos (equivalente al $3 \%$ del total) manifestaron tener síntomas asociados a este nivel. En la Tabla 7 se detallan los porcentajes correspondientes a cada una de las categorías de análisis.

Tabla 7.

Descripción de los indicadores de la escala en el nivel de depresión grave

\begin{tabular}{ccc}
\hline ASPECTO EVALUADO & NO AFECTADO & AFECTADO \\
\hline Tristeza & $0 \%$ & $100 \%$ \\
Pesimismo & $0 \%$ & $100 \%$ \\
Fracaso & $12 \%$ & $88 \%$ \\
Pérdida de placer & $0 \%$ & $100 \%$ \\
Sentimientos de culpa & $0 \%$ & $100 \%$ \\
Sentimiento de castigo & $18 \%$ & $82 \%$ \\
Disconformidad con uno mismo & $0 \%$ & $100 \%$ \\
Autocrítica & $12 \%$ & $88 \%$ \\
Pensamientos o deseos suicidas & $47 \%$ & $53 \%$ \\
Llanto & $18 \%$ & $82 \%$ \\
Agitación & $12 \%$ & $82 \%$ \\
Pérdida de interés & $6 \%$ & $94 \%$ \\
Indecisión & $6 \%$ & $94 \%$ \\
Desvalorización & $6 \%$ & $94 \%$ \\
Pérdida de energía & $6 \%$ & $94 \%$ \\
Cambio de hábitos de sueño & $0 \%$ & $100 \%$ \\
Irritabilidad & $0 \%$ & $100 \%$ \\
Cambios en el apetito & $0 \%$ & $100 \%$ \\
Dificultad de concentración & $0 \%$ & $100 \%$ \\
Cansancio o fatiga & $12 \%$ & $88 \%$ \\
Pérdida de interés en el sexo & $29 \%$ & $71 \%$ \\
\hline & &
\end{tabular}


La Tabla 7 evidencia que en todos los aspectos evaluados en este nivel de depresión se ha determinado afectación en al menos el $80 \%$ de los casos, a excepción de la presencia de deseos suicidas (en 8 personas) y de la pérdida del interés en el sexo (en 11 personas). Se resalta la pérdida del apetito que junto con la reducción en las horas dedicadas a dormir generan aumento del pesimismo, sentimientos de tristeza, culpa e irritabilidad acompañado de reducción en la concentración.

Finalmente, se analizan cada una de las variables demográficas con el nivel de depresión derivado de la aplicación del BDI-2 con el fin de identificar posibles correlaciones entre estas variables. Se recurre a la generación de las tablas cruzadas y a partir de ellas se calcula la Prueba Chi-cuadrado con el fin de validar la hipótesis nula de la independencia entre variables versus la hipótesis alterna de existencia de correlación entre ellas.

Tabla 8.

Análisis de correlaciones entre las variables demográficas y los niveles de depresión experimentado

\begin{tabular}{|c|c|c|c|c|c|c|c|}
\hline \multirow{2}{*}{ VARIABLE } & \multirow{2}{*}{ DIMENSIONES } & \multicolumn{4}{|c|}{ NIVELES DE DEPRESIÓN } & \multirow{2}{*}{$\begin{array}{c}\text { CHI- } \\
\text { CUADRADO }\end{array}$} & \multirow{2}{*}{ CONCLUSIÓN } \\
\hline & & MÍNIMA & LEVE & MODERADA & GRAVE & & \\
\hline \multirow{2}{*}{ Género } & Femenino & $78 \%$ & $15 \%$ & $6 \%$ & $2 \%$ & \multirow{2}{*}{0,018} & \multirow{2}{*}{$\begin{array}{c}\text { Están } \\
\text { correlacionadas }\end{array}$} \\
\hline & Masculino & $73 \%$ & $11 \%$ & $9 \%$ & $7 \%$ & & \\
\hline \multirow{5}{*}{$\begin{array}{l}\text { Rango de edad } \\
\text { (años) }\end{array}$} & Entre 16 y 25 & $66 \%$ & $18 \%$ & $11 \%$ & $5 \%$ & \multirow{5}{*}{0,006} & \multirow{5}{*}{$\begin{array}{c}\text { Están } \\
\text { correlacionada }\end{array}$} \\
\hline & Entre 26 y 35 & $80 \%$ & $11 \%$ & $5 \%$ & $4 \%$ & & \\
\hline & Entre 36 y 45 & $85 \%$ & $13 \%$ & $2 \%$ & $1 \%$ & & \\
\hline & Entre 46 y 55 & $90 \%$ & $10 \%$ & $0 \%$ & $0 \%$ & & \\
\hline & Más de 55 & $76 \%$ & $14 \%$ & $0 \%$ & $0 \%$ & & \\
\hline \multirow{6}{*}{ Estado civil } & Soltero(a) & $68 \%$ & $17 \%$ & $11 \%$ & $5 \%$ & \multirow{6}{*}{0,001} & \multirow{6}{*}{$\begin{array}{c}\text { Están } \\
\text { correlacionadas }\end{array}$} \\
\hline & Casado(a) & $83 \%$ & $13 \%$ & $3 \%$ & $1 \%$ & & \\
\hline & Separado(a) & $79 \%$ & $11 \%$ & $5 \%$ & $5 \%$ & & \\
\hline & Unión libre & $87 \%$ & $10 \%$ & $2 \%$ & $2 \%$ & & \\
\hline & Viudo(a) & $0 \%$ & $0 \%$ & $0 \%$ & $100 \%$ & & \\
\hline & Otra & $75 \%$ & $0 \%$ & $0 \%$ & $25 \%$ & & \\
\hline
\end{tabular}

En la Tabla 8 se registra la posible correlación existente entre los diversos niveles de depresión observados en el grupo de informantes y las variables género, edad y estado civil. Se registran los porcentajes parciales por filas (la sumatoria por filas siempre será el $100 \%$ ), de forma que permite comparar el comportamiento de los diversos niveles de respuesta independientemente del número de personas ubicadas en uno de los niveles de respuesta asociados a cada variable. 
Se puede afirmar que, con respecto al género las mujeres poseen mayores porcentajes en los niveles de depresión mínima y leve, mientras que los hombres las superan en los niveles de depresión moderada y grave, luego se podría afirmar que el aislamiento social obligatorio ha afectado con mayor intensidad a los hombres. Analizando el comportamiento respecto a los diversos grupos etarios, se logra determinar que los niveles de depresión con mayor afectación se concentran entre los 16 y 35 años e independientemente de su estado civil.

\section{DISCUSIÓN}

El estrés, la ansiedad y la depresión son sentimientos que están presentes en los estudiantes que ingresan a la educación superior afectando su salud mental tal como se menciona en las investigaciones de Montoya, Gutiérrez y Toro (2010) y Tijerina et al. (2019). En este sentido la investigación de Rodríguez-Martínez et al. (2018) identificaron que la ansiedad y la depresión son predictores del estrés laboral en docentes de educación superior, presentando mayor afectación en las mujeres. Estos hallazgos son afines con los encontrados en esta investigación dado que se logró determinar que tres de cada cuatro encuestados presentan afectaciones que los ubican en el nivel de depresión mínima la cual se manifiesta en aumento de los sentimientos de tristeza, tensión y cansancio, que se suma al aumento del apetito y pérdida del sueño. Navarro y Dávila (1998) señalan que la falta de sueño suele aparecer en personas con ansiedad generalizada, de este modo los autores afirman que los sujetos con elevada ansiedad tardan más tiempo en quedarse dormidos, presentando menor porcentaje de sueño de ondas lentas, y transiciones más numerosas al sueño ligero.

Con respecto a los hábitos alimenticios, algunos estudios (Páez y Castaño, 2010; Álvarez, Guillén, Ríos y Jiménez, 2011; Chau y Vilela, 2017; Salazar et al. 2016), han determinado que en situaciones de estrés, ansiedad y depresión hay mayor presencia de hábitos alimenticios excesivos y no saludables que sumado a una escasa o leve actividad física pueden desencadenar en sobrepeso, y eso puede afectar el rendimiento académico de los estudiantes (Palacio, Martínez y Tirado, 2006; de Castro, de la Ossa y Eljadue, 2016).

Por otra parte, los resultados del estudio que develan que los sentimientos de tristeza son comunes en escenarios de confinamiento (Berasategi et al., 2020; Andreu, 2020). Sin embargo, es importante aclarar que según trabajos como los de Carranza (2012), la tristeza es un "estado de ánimo pasajero, provocado por una situación concreta pero cuando este estado de ánimo 
perdura en el tiempo, se puede afirmar que se está al frente a depresión moderada o fuerte" (Carranza, 2012, p.89).

Con respecto al género y la prevalencia de mayor depresión en los hombres, Ferrel, Celis y Hernández (2011), manifiestan que el género masculino tiene una tendencia mayor a sufrir depresión grave que el género femenino sobre todo en los estratos más bajos de la sociedad, sin embargo, reafirma que esto depende del programa académico donde realiza sus actividades. Estos resultados son contrarios a los hallazgos de las investigaciones hechas por García et al. (2017) en el que se encontró que las mujeres se ven más afectadas por los sentimientos de tristeza y depresión; o la investigación realizada por Rodríguez y Rodríguez (2017) en dónde el género no resultó tan significativo como si lo fue el programa académico que cursan los estudiantes al determinar la mayor presencia del síndrome de rumiación.

De otro modo, comparando los porcentajes respecto al grupo etario, se puede visualizar que las personas mayores de 46 años presentan síntomas de depresión mínima o leve, en contraste con las personas entre 16 y 45 años quienes están manifestando niveles de depresión moderada o grave, situación que demanda especial atención de las dependencias correspondientes para la implementación de programas de prevención en coordinación y apoyo del grupo familiar en donde actualmente pasa la totalidad de su tiempo el informante, tal como lo resalta la investigación realizada por Barrera-Herrera, Neira-Cofré, Raipán-Gómez Riquelme-Lobos \& Escobar (2019) en la que se concluye que el apoyo social-familiar cumple un rol protector ante los síntomas de depresión, ansiedad y estrés. Estos resultados concuerdan con los trabajos de Bernal (2012), Montesó y Aguilar (2014), Gómez-Romero et al., (2018) y Páez y Peña (2018) quienes afirman que en los últimos años ha aumentado de forma alarmante los casos de depresión en jóvenes universitarios con una peligrosa tendencia al consumo de alcohol, drogas y hasta pensamientos suicidas.

Con relación al estado civil de los encuestados y sus niveles de depresión, se logró determinar que la totalidad de viudos presentan depresión grave, mientras quienes cuya condición es soltero(a), casado(a), separado(a) o unión libre presentan características de depresión mínima principalmente. Estos resultados coinciden con el trabajo de Bastida-González, Valdez-Medina, Valor-Segura, González y Rivera-Aragón, (2017), quienes encontraron que la satisfacción marital es un factor protector de los niveles de depresión. En este sentido Rodríguez et al. (2017) concluyen que la funcionalidad familiar está relacionada con el grado de depresión. 


\section{CONCLUSIONES}

La presente investigación logra evidenciar, en primera instancia, que son escasos los estudios recientes relacionados con el aislamiento masivo en actores educativos debido a virus, epidemias o pandemias. La literatura actual se encuentra publicada en los ámbitos médicos y farmacéuticos. Por esta razón es necesario ampliar investigaciones que indaguen sobre las afectaciones psicológicas de población escolar y de esta manera generar estrategias de intervención para prevenir el aumento de estados depresivos.

Por otra parte, el estudio devela que, hasta la fecha de realización de la encuesta, es decir cuarenta y cinco días después de iniciar el aislamiento obligatorio en Colombia, prevalece la depresión mínima (76\%), seguida de la depresión leve (14\%), la depresión moderada (6\%) y la depresión grave (3\%). Por esta razón se recomienda realizar otro diagnóstico sobre el estado de depresión en comunidades educativas si el aislamiento obligatorio se prolonga, y de esa manera identificar variaciones de estos resultados.

Asimismo, el trabajo muestra que el género masculino tiende a sufrir más de depresión que el femenino posiblemente por el cambio brusco en los hábitos de vida, lo cual podría ser una investigación complementaria a este estudio. Del mismo modo, se resalta que la funcionalidad familiar es un factor decisivo para mitigar el efecto de la depresión. Se resalta que independientemente del nivel de afectación depresivo, se evidenció como aspectos comunes el aumento de los sentimientos de tristeza, tensión, cansancio y del apetito, al tiempo que se observó reducción en la concentración, en los niveles de energía y en las horas de sueño.

Estos resultados evidencian el surgimiento de síntomas depresivos en la totalidad de los informantes posiblemente derivados del aislamiento obligatorio. Este aislamiento ha propiciado cambios en la cotidianidad de las personas, luego se sugiere como una futura investigación que se articule con estos resultados en los que se determine si el desarrollo de los deberes académicos asociados al proceso educativo puede ser un agente generador de estos sentimientos de angustia y depresión.

Por último, es importante aclarar que la generalización de los resultados es limitada debido a que los participantes pertenecen a una facultad de una institución universitaria, pero se suma a la creciente literatura sobre los efectos del aislamiento social propiciado por la pandemia en cotidianidad del 
ser humano. Por lo tanto, se hace necesario comparar este estudio con otras instituciones de características similares.

Conflicto de interés: No existe conflicto de intereses.

Agradecimiento: A los docentes y estudiantes de la Universidad Francisco de Paula Santander participantes del estudio que aceptaron responder los instrumentos.

Financiamiento: Esta investigación se realiza con el aval de los grupos GIPEPP (Grupo de Investigación en Pedagogía y Prácticas Pedagógicas) y GIEZPAZ (Grupo de Investigación en Estudios Sociales y Pedagogía de Paz) de la Universidad Francisco de Paula Santander y financiación propia de los docentes investigadores.

\section{REFERENCIAS}

Almonte, P., Parra, P. \& Baltazar, S. (2019). Prevalencia de signos de depresión y su relación con el desempeño académico en Alumnos de la Universidad Intercultural del Estado de Puebla, México. Holopraxis, 3(1), 140-155. https://www.revistaholopraxis.com/index.php/ojs/article/view/102

Álvarez, G., Guillén, C., Ríos, S. \& Jiménez, M. (2011). Evaluación del estilo de vida y depresión en estudiantes universitarios. Desarrollo Científico Enfermería, 19(8), 264-268. http://www.index-f.com/dce/19pdf/19-264.pdf

Andreu, E. (2020). Actividad física y efectos psicológicos del confinamiento por covid-19. Revista INFAD de Psicología. International Journal of Developmental and Educational Psychology, 2(1), 209-220. https://doi.org/10.17060/ijodaep.2020. n1.v2.1828

Aragón-Nogales R., Vargas-Almanza I. \& Miranda-Novales M. G. (2019). COVID-19 por SARS-CoV-2: la nueva emergencia de salud. Revista Mexicana de Pediatría, 86(6), 213-218. https://doi.org/10.35366/91871

Barrera-Herrera, A., Neira-Cofré, M., Raipán-Gómez, P., Riquelme-Lobos, P., \& Escobar, B. (2019). Apoyo social percibido y factores sociodemográficos en relación con los síntomas de ansiedad, depresión y estrés en universitarios chilenos. Https://doi. org/10.5944/rppc.23676

Bastida-González, R., Valdez-Medina, J. L., Valor-Segura, I., González, N. \& RiveraAragón, S. (2017). Satisfacción marital y estado civil como factores protectores de la depresión y ansiedad. Revista Argentina de Clínica Psicológica, 26(1), 95-102. https://doi.org/10.24205/03276716.2017.1008

Beck, A., Steer, R., \& Brown, G. (1996). BDI-Il. Beck Depression Inventory-Second Edition manual. San Antonio, Texas: The Psychological Corporation.

Beck, A., Steer, R., \& Brown, G. (2009). BDI-II. Inventario de Depresión de Beck. Segunda Edición Manual. Buenos Aires: Paidós.

Berasategi, N., Idoiaga, N., Dosil, M., Eiguren, A., Pikatza, N. \& Ozamiz, N. (2020). Las voces de los niños y de las niñas en situación de confinamiento por el COVID-19. Bilbao: Universidad del País Vasco. https://web-argitalpena.adm.ehu.es/pdf/ USP00202291.pdf

Bernal, J. (2012). Depresión y consumo de alcohol en jóvenes universitarios (Análisis investigativo, Institución Universitaria Politécnico Grancolombiano). Repositorio Institucional Alejandría. http://alejandria.poligran.edu.co/handle/10823/846 
Burgos, G. (2014). Proyecto Vive la vida: Propuesta de intervención basada en un programa de psicología positiva y motivacional para mejorar la autoestima y disminuir el aislamiento social en mayores jubilados (Trabajo Final de Grado). Universitat Oberta Catalunya, Barcelona, España. http://openaccess.uoc.edu/ webapps/o2/bitstream/10609/41021/5/gburgosgTFG1214memoria.pdf

Carranza, R. (2012). Depresión y características demográficas asociados en estudiantes y líderes universitarios de Lima metropolitana. Apuntes Universitarios, 2(2). https://doi.org/10.17162/au.v0i2.298

Chau, C. \& Vilela, P. (2017). Determinantes de la salud mental en estudiantes universitarios de Lima y Huánuco. Revista de Psicología (PUCP), 35(2), 387-422. https:// dx.doi.org/10.18800/psico.201702.001

De Castro, A., De la Ossa, J. \& Eljadue, A. (2016). Experiencia de ansiedad desde la perspectiva humanista existencial en estudiantes universitarios de Cali y Cartagena. Itinerario Educativo, 30(68), 79-94. https://doi.org/10.21500/01212753.2949

Falcón, V., Pertile, V. \& Ponce, B. (2019). La encuesta como instrumento de recolección de datos sociales: Resultados diagnostico para la intervención en el Barrio Paloma de la Paz (La Olla) - ciudad de Corrientes (2017-2018). En Construyendo una Geografía Crítica y Transformadora: En defensa de la Ciencia y la Universidad Pública [Actas]. Ensenada, Argentina: Universidad Nacional de La Plata. http:// www.memoria.fahce.unlp.edu.ar/trab_eventos/ev.13544/ev.13544.pdf

Ferrel, R., Celis, A. \& Hernández, O. (2011). Depresión y factores socio demográficos asociados en estudiantes universitarios de ciencias de la salud de una universidad pública (Colombia). Psicología desde el Caribe, 27, 40-60. http://rcientificas. uninorte.edu.co/index.php/psicologia/article/viewFile/3076/2129

Gamboa, A. (2016). Docencia, investigación y gestión: Reflexiones sobre su papel en la calidad de la educación superior. Revista Perspectivas, 1(1), 81-90. https://doi. org/10.22463/25909215.973

García, R., Valencia, I., Hernández-Martínez, A. \& Rocha, T. (2017). Pensamiento rumiativo y depression entre estudiantes universitarios: Repensando el impacto del género. Revista Interamericana de Psicología/Interamerican Journal of Psychology, 51(3), 406-416. https://doi.org/10.30849/rip/ijp.v51i3.384

Gómez-Romero, M., Limonero, J., Toro, J., Montes-Hidalgo, J., \& Tomás-Sábado, J. (2018). Relación entre inteligencia emocional, afecto negativo y riesgo suicida en jóvenes universitarios. Ansiedad y Estrés, 24(1), 18-23. https://doi.org/10.1016/j. anyes.2017.10.007

Gorbalenya, A., Baker, S., Baric, R., De Groot, R., Drosten, C., Gulyaeva, A., Haagmans, B., Lauber, C., Leontovich, A., Neuman, B., Penzar, D., Perlman, S., Poon, L., Samborskiy, D., Sidorov, I., Sola, I., \& Ziebuhr, J. (2020). Severe acute respiratory syndrome-related coronavirus-The species and its viruses, a statement of the Coronavirus Study Group. BioRxiv. https://doi.org/10.1101/2020.02.07.937862

Guzmán-Del Giudice, O., Lucchesi-Vásquez, E., Trelles-De Belaúnde, M., Pinedo-Gonzales, R., Camere-Torrealva, M., Daly, A. \& Pichilingue-Chagray, J. (2020). Características clínicas y epidemiológicas de 25 casos de COVID-19 atendidos en la Clínica Delgado de Lima. Revista de la Sociedad Peruana de Medicina Interna, 33(1), 15-24. https://doi.org/10.36393/spmi.v33i1.506

Instituto Nacional de Salud. (2020). Covid-19. https://infogram.com/covid-19-or-instituto-nacional-de-salud-or-colombia-1hke60w3qlz345r

Lara, T. (2009). El papel de la Universidad en la construcción de su identidad digital. RUSC. Universities and Knowledge Society Journal, 6(1). http://dx.doi. org/10.7238/rusc.v6i1.25 
Martín, M., Hernández, C. \& Mendoza, S. (2017). Ambientes de aprendizaje basados en herramientas web para el desarrollo de competencias TIC en la docencia. Revista Perspectivas, 2(1), 97-104. https://doi.org/10.22463/25909215.1282

Montesó-Curto, P. \& Aguilar-Martín, C. (2014). Depresión según la edad y el género: análisis en una comunidad. Atención Primaria, 46(3), 167. http://dx.doi. org/10.1016/j.aprim.2013.07.009

Montoya, L., Gutiérrez, J. \& Toro, B. (2010). Depresión en estudiantes universitarios y su asociación con el estrés académico. CES Medicina, 24(1). https://revistas.ces. edu.co/index.php/medicina/article/view/1011

Navarro, J. \& Dávila, G. (1998). Psicopatología y sueño. II. Trastornos por ansiedad. Psicología Conductual, 6(1), 103-114. https://www.behavioralpsycho. com/wp-content/uploads/2020/04/Download-contents-Behavioral-Psychology-1993-2020.pdf

Organización Mundial de la Salud (2020). COVID-19: cronología de la actuación de la OMS. https://www.who.int/es/news-room/detail/08-04-2020-who-timeline--covid-19

Ovalles, G., Urbina, J. \& Gamboa, A. (2014). Abandono y permanencia: factores pedagógicos en educación superior. Bogotá: Ecoe Ediciones.

Pabón-Poches, D. (2019). Riesgo de maltrato en personas adultas mayores: validación de contenido de una escala. Psicogente, 22(41), 1-27. https://doi.org/10.17081/ psico.22.41.3299

Páez, M. \& Castaño, J. (2010). Estilos de vida y salud en estudiantes de una facultad de psicología. Psicología desde el Caribe, (25), 155-178. http://rcientificas.uninorte. edu.co/index.php/psicologia/article/view/42

Páez, M. \& Peña, F. (2018). Depresión en universitarios. Diversas conceptualizaciones y necesidad de intervenir desde una perspectiva compleja. Archivos de Medicina, 18(2), 339-351. https://doi.org/10.30554/archmed.18.2.2747.2018

Palacio, J., Martínez, Y., Ochoa, N., \& Tirado, E. (2006). Relación del rendimiento académico con las aptitudes mentales, salud mental, autoestima y relaciones de amistad en jóvenes universitarios de atlántico y bolívar. Psicogente, 9(15), 11-31. http://revistas.unisimon.edu.co/index.php/psicogente/article/view/2664

Prada, R., Hernández, C. \& Gamboa, A. (2019a). Different scenarios for the teaching of mathematics with the support of virtual platforms: Flipped classroom. Journal of Physics: Conference Series, 1388(1), 1-5. https://iopscience.iop.org/ article/10.1088/1742-6596/1388/1/012046

Prada, R., Hernández, C. \& Gamboa, A. (2019b). Usos y efectos de la implementación de una plataforma digital en el proceso de enseñanza de futuros docentes en matemáticas. Revista Virtual Universidad Católica del Norte, (57), 137-156. https://doi.org/10.35575/rvucn.n57a10

Rama, C. (2019). Las dinámicas del poder en la educación a distancia y virtual. Cuadernos Universitarios, 12(12), 37-50. http://revistas.ucasal.edu.ar/index.php/ CU/article/view/266

Rangel, A. \& Peñalosa, E. (2013). Alfabetización digital en docentes de educación superior: construcción y prueba empírica de un instrumento de evaluación. Píxel-Bit. Revista de Medios y Educación, 0(43), 9-23. http://dx.doi.org/10.12795/ pixelbit.2013.i43.01

Ríos-González, C. (2019). Implicancias del COVID-19, una nueva enfermedad producida por coronavirus. Medicina Clínica y Social, 3(3), 71-72. https://www. medicinaclinicaysocial.org/index.php/MCS/article/view/108/77 
Rodríguez, A., Haro, M., Martínez, R., Ayala, R. \& Román, A. (2017). Funcionalidad familiar y depresión en adultos en la atención primaria. RESPYN Revista Salud Pública y Nutrición, 16(4), 20-23. https://doi.org/10.29105/respyn16.4-3

Rodríguez, S. \& Rodríguez, M. (2017). Estrategias cognitivas de regulación emocional y síntomas de depresión en estudiantes universitarios: diferencias por género y tipo de carrera. Resultados preliminares. Revista Akadèmeia, 16(1), 113-130. http://revistas.ugm.cl/index.php/rakad/article/view/147

Rodríguez-Martínez, M., Tovalin-Ahumada, J. H., Gil-Monte, P. R., Salvador-Cruz, J. \& Acle-Tomasini, G. (2018). Trabajo emocional y estresores laborales como predictores de ansiedad y depresión en profesores universitarios mexicanos. Información psicológica, (115), 93-106. http://dx.medra.org/10.14635/IPSIC.2018.115.11

Ruiz-Bravo, A. \& Jimenez-Varela, M. (2020). SARS-CoV-2 y pandemia de síndrome respiratorio agudo (COVID-19). Ars Pharmaceutica (Internet), 61(2), 63-79. http:// dx.doi.org/10.30827/ars.v61i2.15177

Salazar, D., Castillo, T., Pastor, M., Tejada-Tayabas, L. \& Palos, A. (2016). Ansiedad, depresión y actividad física asociados a sobrepeso/obesidad en estudiantes de dos universidades mexicanas. Hacia la promoción de la salud, 21(2), 99-113. http://dx.doi.org/10.17151/hpsal.2016.21.2.8

Sánchez, Á., Ramírez, B. \& Cañón, N. (2019). Retos de las políticas educativas de educación superior: una mirada inclusiva de la educación rural en perspectiva de las nuevas competencias digitales. En Novoa, A. \& Ramírez-Orozco, M. (comp.). La educación y el sujeto político: Aporte crítico. Ediciones Unisalle

Stefani, D., Seidmann, S., Pano, C., Acrich, L., \& Bail, V. (2003). Los cuidadores familiares de enfermos crónicos: sentimiento de soledad, aislamiento social y estilos de afrontamiento. Revista Latinoamericana de Psicología, 35(1),55-65. https://www.redalyc.org/articulo.oa?id=805/80535105

Tijerina, L., González, E., Gómez, M., Cisneros, M., Rodríguez, K. \& Ramos, E. (2019). Depresión, ansiedad y estrés en estudiantes de nuevo ingreso a la educación superior. RESPYN Revista Salud Pública y Nutrición, 17(4), 41-47. https://doi. org/10.29105/respyn17.4-5

Esta obra está bajo: Creative commons attribution 4.0 international license. El beneficiario de la licencia tiene el derecho de copiar, distribuir, exhibir y representar la obra y hacer obras derivadas siempre y cuando reconozca y cite la obra de la forma especificada por el autor o el licenciante.

\section{(cc) BY}

